\title{
WHAT IS A DIGITAL TWIN? - DEFINITIONS AND INSIGHTS FROM AN INDUSTRIAL CASE STUDY IN TECHNICAL PRODUCT DEVELOPMENT
}

\author{
J. Trauer ${ }^{1, \otimes}$, S. Schweigert-Recksiek ${ }^{1}$, C. Engel ${ }^{2}$, K. Spreitzer ${ }^{2}$ and \\ M. Zimmermann ${ }^{1}$ \\ ${ }^{1}$ Technical University of Munich, Germany, ${ }^{2}$ Viessmann Werke GmbH \& Co. KG, Germany \\ $\bowtie$ jakob.trauer@tum.de
}

\begin{abstract}
Over the last two decades, a concept called Digital Twin has evolved rapidly. Yet, there is no unified definition of the term. Based on a literature study and an industrial case study, an overarching definition of Digital twins is presented. Three characteristics were identified - representation of a physical system, bidirectional data exchange, and the connection along the entire lifecycle. Further, three sub-concepts are presented, namely: Engineering Twin, Production Twin, and Operation Twin. The presented paper thus formulates a consistent and detailed definition of Digital Twins.
\end{abstract}

Keywords: digital twin, product development, virtual engineering (VE), product lifecycle management (PLM), industry 4.0

\section{Introduction}

In the last decade, companies of all sizes, all around the globe are facing ever more fast-paced, uncertain, and complex boundary conditions. A driver of this phenomenon is the growing digitization forcing companies to develop more cost- and time-efficiently. On the other hand, digital or virtual engineering also enables companies to cope with these challenges (Hanschke, 2018). In the course of this trend, a theory called Digital Twin has developed over the last two decades. The term describes the virtual representation of a physical system. In the beginning, Digital Twins were merely descriptive, but as computational and information and communication technologies evolved, it became possible to establish a bidirectional coupling between the digital and the physical system (Grieves and Vickers, 2017). Since its basic idea in 2002, the relevance of Digital Twins has grown increasingly. In a workshop with 40 Swedish industrial and academic experts, Eckert et al. (2019) identified "complete integrated twins" as one of the major industry trends to 2040. The expected benefits are manifold. According to Jones et al. (2019), the benefits of "twinning" concepts lie in an increased accuracy and fidelity as well as decreased time/costs and work load in the process of creating real-time virtual representation or in the process of realising virtual descriptions in the physical system. However, according to Tao et al. (2019) to date, Digital Twins were primarily applied in production or afterproduction stages, rarely in earlier stages such as product design. Despite the large number of occurrences in literature, there is still no consistent, overarching definition of Digital Twins.

The results presented in this contribution were elaborated in an industrial case study with the intention of developing a comprehensive approach supporting companies in the conception and implementation of Digital Twins. The corresponding case study is described in the paper of Schweigert-Recksiek et al. (2020). 


\section{Methodology}

\subsection{Industrial case study}

The case study partner of this contribution is Viessmann, an internationally active company in the field of heating technology. The German, medium-sized company with more than 10,000 employees develops and produces heating technology products for residential and commercial applications. The case study partner is increasingly confronted with the introduction of digital technologies, accelerated changes in customer needs, the collection and use of data, and shortened innovation and product life cycles. Thus, the company is currently undergoing an extensive process of change, which is primarily the result of the ongoing digitization. In the course of this transformation, a case study was initiated on the conception and initial implementation of a Digital Twin. The developed procedure model is presented in Schweigert-Recksiek et al. (2020).

\subsection{Systematic literature review}

In the beginning of the collaboration with the industry partner, it was observed from an initial literature review that there is an insufficient basis of definitions on Digital Twins. Kritzinger et al. (2018) had the same impression from literature on Digital Twins in manufacturing. Out of over 40 publications, only $5 \%$ included definitions of the term. Among these publications, they did not find a common definition. Therefore, it was decided to choose type 2 - "Comprehensive Study of the Existing Situation" - of the Design Research Methodology by Blessing and Chakrabarti (2009) for the research of this paper. Based on a literature-based research clarification, a comprehensive descriptive study was conducted, followed by an initial prescriptive study in the form of short discussion. To initiate and structure the comprehensive literature review on the state of art and research of Digital Twins, a research strategy was developed as shown in Figure 1.

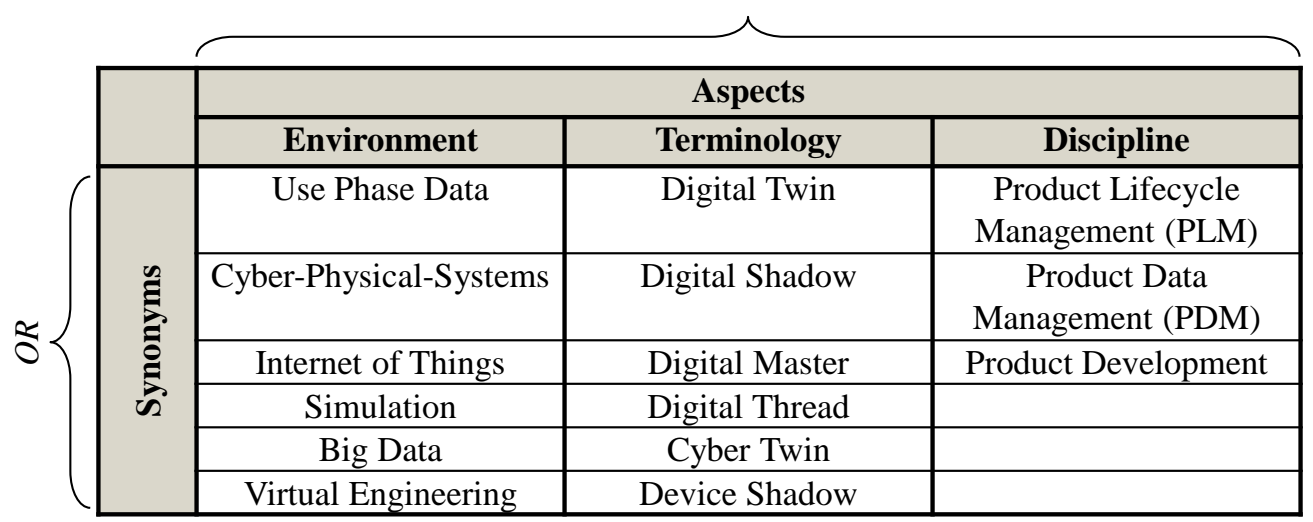

Figure 1. Research strategy for the literature review on the state of art of Digital Twins

These keywords were entered in Scopus (latest search request December 2019). For each search request, one synonym of each aspect was combined using a Boolean "AND" operator. In a second step, the search requests were aggregated by using an "OR" operator. Results based on these 15 factors were systematically limited to the field of engineering, publications containing Digital Twin as a Keyword, and finally filtered by their content. The procedure of the keyword search process is depicted in Figure 2.

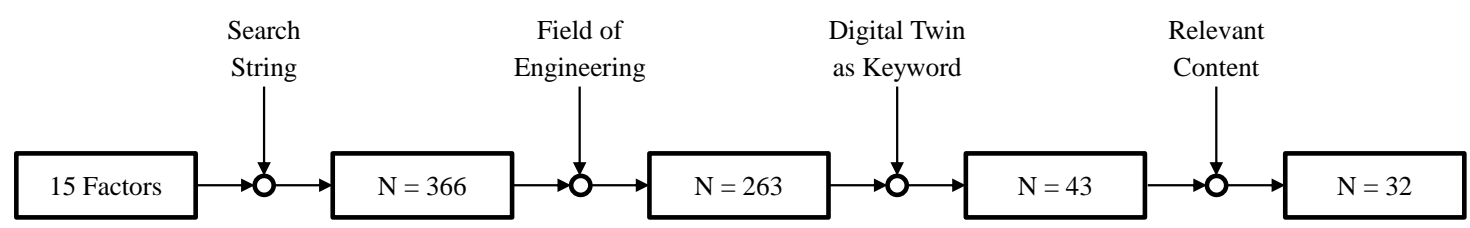

Figure 2. Keyword search process

Additional publications were identified through targeted consultation of textbooks and journals as well as tracing references from identified literature. In total, the authors examined 57 publications. 


\section{Literature review}

\subsection{Early definitions}

The concept of a Digital Twin was first mentioned in a presentation of the University of Michigan in 2002 entitled "Conceptual Ideal for PLM" (Grieves and Vickers, 2017). Even though it referred to Product Lifecycle Management, the presented concept contained basic characteristics of Digital Twins that have not significantly changed since then - a "real space" and a "virtual space" connected via data and information exchange (Grieves and Vickers, 2017). A few years later in 2010 the term Digital Twin appeared in the most widely used definition of the NASA as an "integrated multi-physics, multiscale, probabilistic simulation of a vehicle or system that uses the best available physical models, sensor updates, fleet history, etc., to mirror the life of its corresponding flying twin." (Shafto et al., 2010). A more current definition in manufacturing defines the term as "...a digital representation of an active unique product [...] or unique product-service system [...] that comprises its selected characteristics, properties, conditions, and behaviours by means of models, information, and data within a single or even across multiple life cycle phases. " (Stark and Damerau, 2019).

\subsection{Scope of digital twins}

Closely related to the many definitions of Digital Twins, related terms used as synonyms arose. Referring to NASA's definition, the most important characteristic of a Digital Twin was creating a most accurate virtual representation of the real physical product. Most publications stick to this definition, aiming at an increased convergence between real and virtual space (Boschert and Rosen, 2016; Grieves and Vickers, 2017; Cadet et al., 2017; Stark et al., 2018). Even though most publications agree on the virtual space, they differ regarding the real space. Some refer to machines, or more generally to products (Baltes and Freyth, 2017; Boureanu, 2017; Tao et al., 2019). Some consider the assets of a company as the real space (Kasote et al., 2017; Banerjee et al., 2017; Stark et al., 2017). Others, e.g. Lee et al. (2017), extend the notion even further and include whole systems in their consideration. According to Kuhn (2017), apart from physical products a Twin can also represent non-physical "things" such as services.

Regardless of the represented artefact, it needs to be defined which aspects of the real space should be transferred to the virtual space. The disunity in literature on how to model the behaviour is even bigger here. Some come from a PLM point of view, representing the physical artefact based on productrelated data, such as the bill of materials (e.g. Lee et al., 2017). Others try copying the physical behaviour, its properties, and situations to model the artefact's current status (Shafto et al., 2010; Baltes and Freyth, 2017; Boureanu, 2017; Yun et al., 2017). Some authors pledge for a comprehensive representation of all information and knowledge of the physical twin in the Digital Twin (Schroeder et al., 2016). Tao et al. (2019) go even further by considering not only data generated by the physical product, but also data that is generated due to integration, fusion, and analysis of data from both spaces. In contrast, Boschert and Rosen (2016) state that such a vast amount of data and information should be avoided. Instead, it should only contain the data necessary and relevant for the use-casespecific applications in present and future lifecycle phases. Also in the definition of Stark and Damerau (2019) only selected properties, conditions, etc. should be transmitted. Riesener et al. (2019) present an approach for selecting relevant data. However, the selected information should consider all perspectives of a product, including customers, product structure, functions, requirements, geometrics, technology, production, and finances. Schleich et al. (2017) pledge for implementing an abstract conceptual model and a virtual representation. This differentiation makes the model scalable, interoperable, expansible, and enables it to achieve high fidelity.

A Digital Twin is not just defined by the included data - it also includes algorithms, mostly simulations (Kuhn, 2017; Boschert and Rosen, 2016). Consequently, a Digital Twin is often described as the next level simulation as it includes real use phase data (Boschert and Rosen, 2016; Schluse and Rossmann, 2016).

The discussion about data to be included and about the lifecycle of a Digital Twin, which is outlined in the next section, is closely related to the Twin's connection to the real space. Here, two groups can be 
found in literature. One describing a Digital Twin as a self-sufficient virtual image, thus there is no exchange of information between the virtual and the real space (Schluse and Rossmann, 2016; Bajaj et al., 2016). The other group regards the need to exchange information between the two spaces (Huber and Kaiser, 2015; Baltes and Freyth, 2017; Shafto et al., 2010; Schleich et al., 2017). This information can be both unidirectional and bidirectional (Baltes and Freyth, 2017; Yun et al., 2017; Boschert et al., 2018).

The understanding of Digital Twins also in industrial applications varies. General Electrics for example implements health monitoring by collecting and analysing data of their products over the lifecycle. Siemens instead aims at improving efficiency and quality of their manufacturing systems by digitizing their production in the context of Industry 4.0 (Schleich et al., 2017). To conclude, there are unlimited aspects, that can or could be implemented and they highly depend on the use case scenario. Literature agrees in the understanding that a Digital Twin is based on certain virtual artefacts. Interestingly there are two directions in the state of research - one focussing on the simulation aspect (e.g. Boschert and Rosen, 2016; Schluse and Rossmann, 2016; Shafto et al., 2010), the other rather on the virtual representation based on a data basis (e.g. Huber and Kaiser, 2015; Lee et al., 2017; Schroeder et al., 2016).

\subsection{Digital twins in product lifecycle context}

As the concept was emerging out of the field of PLM, a core characteristic of a Digital Twin is the consideration of lifecycle phases. In his first presentation in 2002, Grieves referred to the connection between real space and virtual space over all phases of the product lifecycle (Grieves and Vickers, 2017). Stark and Damerau (2019) do not consider the whole lifecycle. In their definition, the twin can exist during single, or multiple lifecycle phases. Cadet et al. (2017) specify the lifecycle from engineering over production to operation. Starting point and end point of the Digital Twin are not defined. Schroeder et al. (2016) particularize the timespan and suggest the existence of a Twin from the beginning of a product's life until its deposal. Tao et al. (2019) suggest to model all phases including e.g. design, manufacturing, quality, maintenance, repair, and overhaul. In some publications a Digital Twin can exist earlier than the physical product (Shafto et al., 2010; Grieves and Vickers, 2017; Lee et al., 2017; Eigner et al., 2019). For example, according to |Kuhn (2017), a production plant that is still in the planning process can already have a Digital Twin describing the future characteristics of that plant. It could be applied as well to a product, which is not yet produced. According to Eigner et al. (2019), a Digital Twin exists over the complete lifecycle, subdivided in the phases "as-designed", "as-built", and "as-maintained". The starting point in early phases of the product development process is also in line with the definition of Boschert and Rosen (2016), as the Digital Twin can already exist in the form of virtual models, simulations, and their connection.

\subsection{Related terms}

Despite the divergent understanding on the characteristics of a Digital Twin, there are also other terms, which are partially used as synonyms. Beneath more uncommon notions, such as "Digital Blueprint" (e.g. Bajaj et al., 2016), "Digital Mirror Model" (e.g. Erikstad, 2017), "Digital Reflection" (e.g. Erikstad, 2017), or "Dynamic Data Driven Application System" (e.g. Bazilevs et al., 2015), the most widely used are "Digital Thread" (e.g. Siedlak et al., 2018) and "Device/Digital Shadow" (e.g. Schuh and Blum, 2016; Baltes and Freyth, 2017). In a literature review, Riesener et al. (2019) identified 21 publications from 2011 to 2019 using one or more of the three terms. Only five of them considered solely Digital Twins, four used the term Digital Thread, five the term Digital Shadow. The remaining seven publications used a combination of at least two of the terms.

Referring to Lubell et al. (2013) and Helu and Hedberg (2015), a Digital Thread is the continuous connection of all digital models over the entire product lifecycle phases, enabling a traceability from requirements until retirement. According to Siedlak et al. (2018), a Digital Thread refers to the connection of digital models from different disciplines with the goal of reducing design time and enabling interdisciplinary information exchange to overcome organizational boundaries. Boschert and Rosen (2016) and West and Blackburn (2017) differentiate the Twin from the Thread by the considered lifecycle phases - while the Twin focusses on phases of operation and service, the Thread addresses earlier phases of acquisition and design. The term Digital Shadow is often used synonymously 
(Schuh and Blum, 2016; Schroeder et al., 2016). The German Society for Production Engineering instead differentiates the Shadow from the Twin as the Shadow is just a sufficiently precise representation of the real space (Bauernhansl et al., 2016). The Shadow projects the real space to the virtual space so that consequently the Digital Twin can create a most accurate representation of the real space. Kritzinger et al. (2018) differentiate between the terms "Digital Shadow" and "Digital Twin" based on the level of data integration. The lowest level is a "Digital Model", representing an existing or planned physical product in the virtual space. However, they are just connected through a manual data exchange, so a change in the virtual or real space does not directly affect the other space. The Shadow only differs from the Digital Model, as the physical object is connected automatically with the virtual representation. Thus, if the product changes, the virtual representation will adapt automatically. The Digital Twin is on the highest level of connectivity, connecting both spaces automatically. Therefore, the virtual model could take for example a controlling function on the product and directly change the physical object.

\section{Definition and types of Digital Twins}

The terms Digital Shadow, Thread, and Twin were also discussed within the industrial case study this paper builds on. As a result, there was an overall accordance that these terms are not interchangeable . A Digital Model is considered to be a mere virtual representation of a physical object, but without any interconnectivity between the real and the virtual space. The word Shadow was classified as unsuitable as a Shadow is just an image of reality with rudimentary properties. Moreover, a Shadow cannot give feedback to the real system, so a bidirectional data exchange is not possible. These associations align to some extend with literature as the Shadow is often described as a partial implementation of a Twin or a pre-step towards a Twin (e.g. Bauernhansl et al., 2016). The industry partner describes a Digital Thread as the "digital coupling of all relevant information within the product lifecycle". In contrast to the Digital Shadow, it can exist along with the Twin and even more: it is most beneficial together with a Twin. The Twin differs from the Thread by behaving as realistic as possible and by exchanging information with its physical twin in an automated and bidirectional way. Therefore, in line with e.g. Kritzinger et al. (2018), a hierarchy of the mentioned terms is suggested - from a Digital Model, over a Digital Thread the Digital Shadow, to the Digital Twin. Each concept entails a higher fidelity and enables more automated bidirectional data exchange than the previous one.

Based on the previously described findings from the literature review and the insights from the case study partner, there are three crucial characteristics of Digital Twins, which should be included in the definition:

1. The Digital Twin is a virtual dynamic representation of a physical artefact or system,

2. Data is automatically and bidirectionally exchanged between the Digital Twin and the physical system,

3. The Twin entails data of all phases of the entire product lifecycle and is connected to all of them.

Consequently, the following definition was derived in accordance with the industry partner:

\section{A Digital Twin is a virtual dynamic representation of a physical system, which is connected to it over the entire lifecycle for bidirectional data exchange.}

The physical twin automatically transfers, amongst others, data of its behaviour, its status, and information on the environment from the real space to the virtual space over the entire product lifecycle, when needed. The virtual twin instead identifies product or process oriented improvements, control demands based on the current situation, or predictions of the near future and sends them back to the real space so the physical product adapts accordingly. This data exchange happens automatically (cf. Figure 3). Here, it is important to differentiate between data and information. The twins only exchange data. Information is only generated in the real, respectively the virtual space based on the transmitted data. In the presented definition, in line with the findings of Kritzinger et al. (2018), an automatic data exchange is required. It is not meant that there is a constant data transmission. Data should only be transferred on demand, so that the Digital Twin can represent the Physical Twins 
sufficiently well, respectively when the Digital Twin needs to influence the physical object in the real space. Regarding the amount of data, only data required for the respective use case should be entailed.

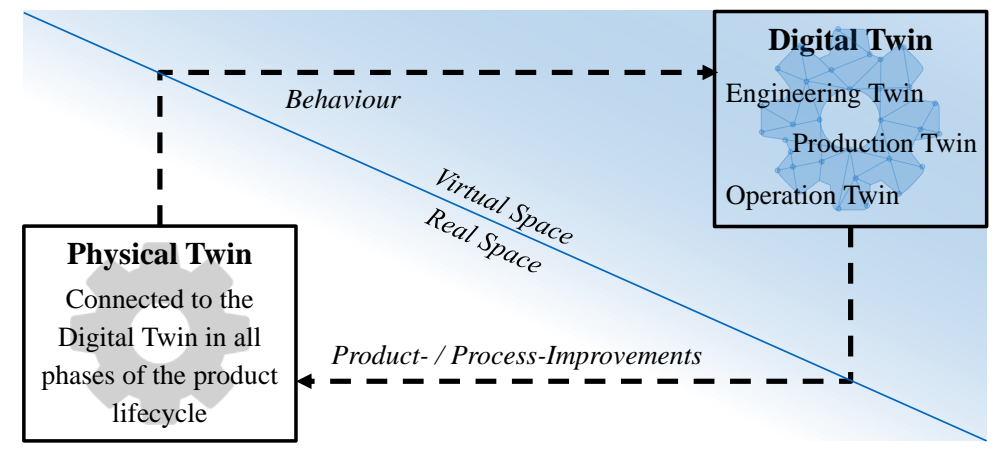

Figure 3. Concept of a Digital Twin

The presented definition unifies the previously identified and highlighted aspects of Digital Twins. However, it is quite broad and generic. Consequently, there was a need to further detail the concept or to allow for subdimensions. Thus, in accordance with the case study partner, subdimensions of the term Digital Twin were defined. As the concept evolved from the field of PLM, it was decided that the most helpful way would be a breakdown of the term according to the phases of the product lifecycle. This is also in line with parts of the literature (e.g. Eigner et al., 2019). Thus, the definition was split into three subtypes of Digital Twins - the Production Twin, the Engineering Twin, and the Operation Twin (cf. Figure 3).

When defining Digital Twins, it should be highlighted that there is no specific example for Digital Twins. A Digital Twin is rather defined by a collection of use cases contributing to an overall strategy with a vision aligning to the above-mentioned definition. Consequently, a Digital Twin is always a collection of different use case scenarios and it is not purposeful limiting the twinning principles to just some specific applications. Thus, in the case study, the developed subtypes were also specified based on use cases. Use cases that contribute to the purpose of the respective phase are consequently part of that specific twin. Typical, promising use cases were collected in order to explain the difference between the individual subtypes and to assess their potential. However, they are not always completely separable and might contribute to different phases. This exemplary overview of use cases is shown in Figure 4. It gives an overview of possible use cases, however, it is a highly companyspecific collection. This overview should also help to concretize the high-level definition. Numerous other examples could be used for defining the sub-types. Nevertheless, they are similar for instance to the investigations of Lim on PLM advantages by Digital Twins.

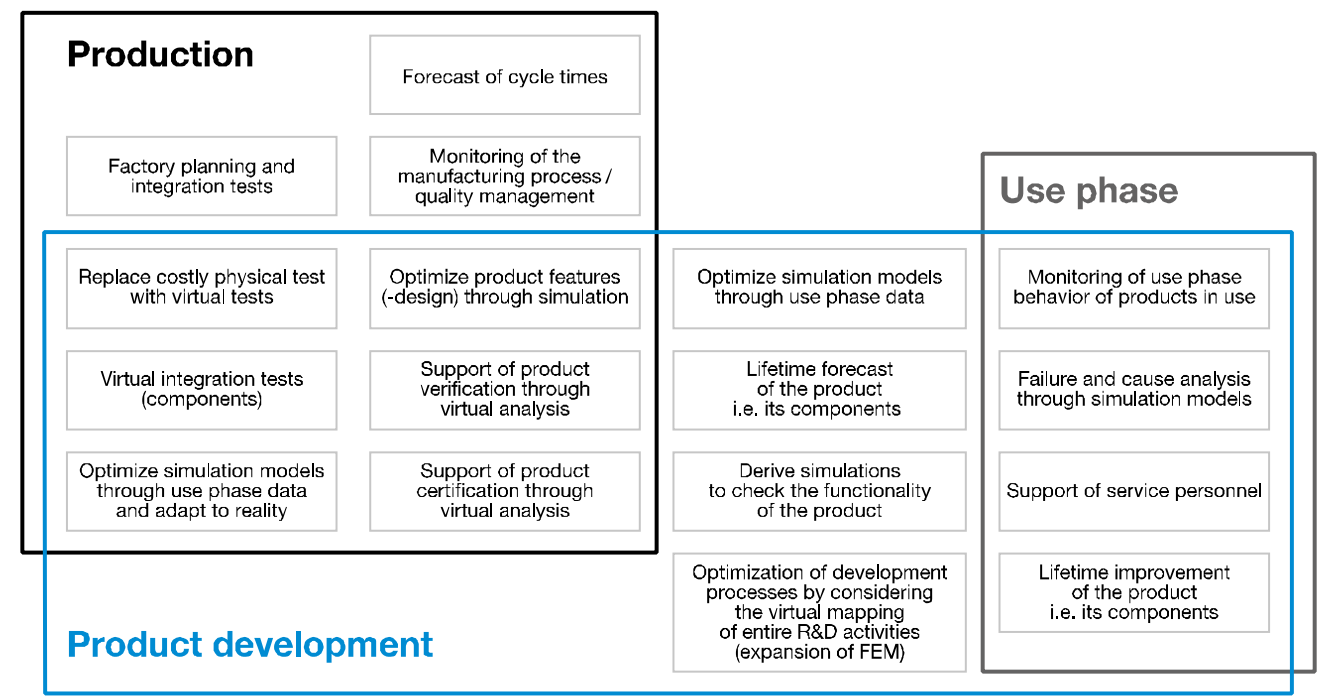

Figure 4. Exemplary use cases for Digital Twins 
The previously presented definition demands the connection of the physical and virtual space over the entire product lifecycle. However, it might seem that there is a conflict as a physical system is not yet available in early phases of the product lifecycle. This would especially collide with the definition of an Engineering Twin. However, the authors explicitly include these phases, where no physical product exists yet as long as they contribute to an overall twinning strategy. This means a physical product should be available in a foreseeable time and combined with other planned use cases, all characteristics are fulfilled. To support this strategy-based definition, a shell model for the conception and implementation of a Digital Twin was developed (cf. Figure 5). It consists of several levels on which use cases can be implemented. Use cases on the innermost shell would not fully align with our definition of a Digital Twin. With each shell, the level of complexity regarding the source of use phase data and the virtual model rises. The stages are gradually filled until the Digital Twin is able to represent and interact with the physical product over the entire lifecycle. In the underlying case study, first use cases were chosen entailing only use cases that include data from the test bench, but not from the entire device. Over the next few years, the twinning concept will be expanded to include further use cases so that data from production, use, maintenance, and disposal can gradually be used as well.

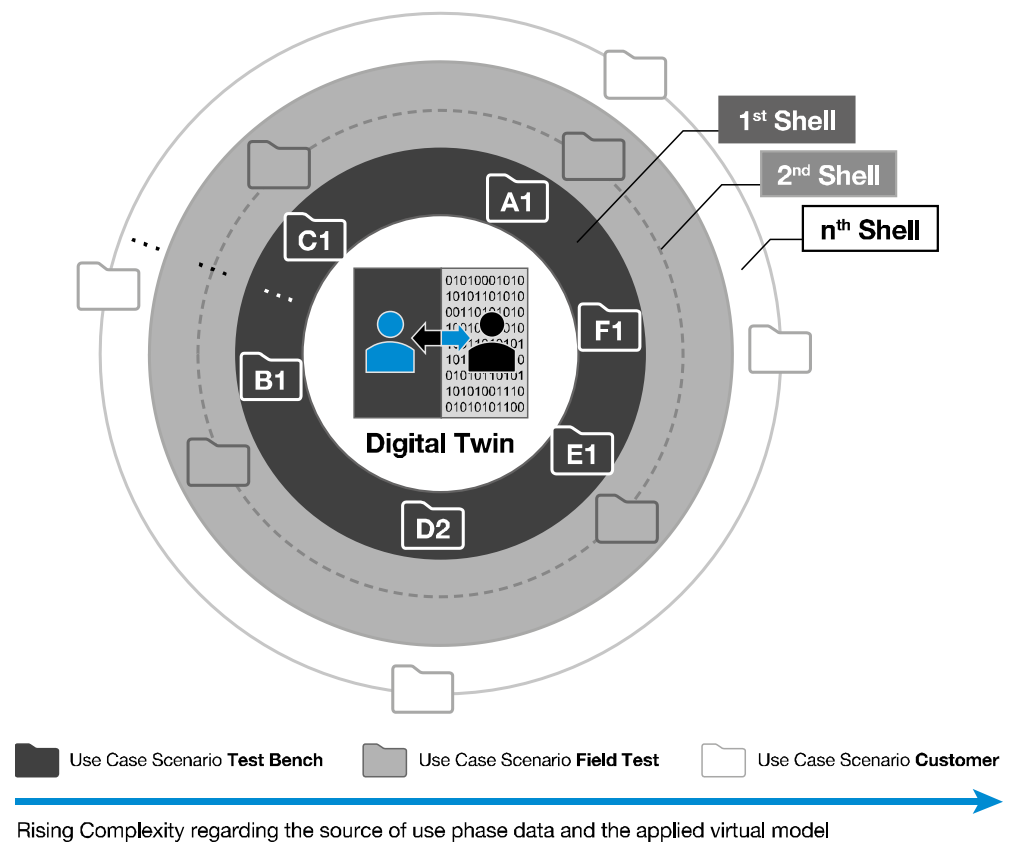

Figure 5. Shell model for the conception and implementation of Digital Twins

\section{Discussion and outlook}

The paper presents a consistent and overarching definition for Digital Twins based on a systematic literature review and the results of an industrial case study. Of course, this definition also has its limitations and it does not claim to be solely valid. However, in the course of the collaboration with the industry partner, this will be the underlying definition for the further implementation of the twin concept. Thus, this paper presents the basis for the case study of Schweigert-Recksiek et al. (2020).

The authors perceive the greatest limitation of the definition in the restriction to "physical products" in the real space. Thus, it would be impossible to call the concept a Digital Twin as long as the physical pendant is not yet manufactured or existing. However, as it was previously outlined, the Digital Twin consists of several use cases and is more of a whole strategy than just a single instance. This is also in line with most of the studies identified in the literature review (e.g. Schleich et al., 2017; Jones et al., 2019). Consequently, the authors of this paper consider the combination of a not yet existing physical entity in the real space as a Digital Twin, as long as a physical product will be available in the near future and the use case contributes to the overall twinning strategy.

Overall, the definition may seem quite strict and narrow. It was formulated like this on purpose as it should serve as an ambitious vision for Digital Twins, describing the highest possible maturity of this 
technology. However, the authors are aware that Digital Twins need to evolve over time to achieve this maturity level. Thus, preliminary versions can also be considered as a Digital Twin, even though they might not yet fulfil all elements of the definition.

One of the key characteristics is that the Digital Twin is able to affect the physical twin directly using simulation, predictions, etc. This is especially the case in the engineering and production phase. In the operational phase, there are also use cases where the Digital Twin affects the real space, but only few. More often, the information of the Digital Twin can be used to adapt future products, for example in the context of Data-Driven Engineering (Trauer et al., 2020).

By defining Digital Twins based on use cases, it is possible to cover a broad range of possible applications. Further, it enables the consistent definition of possible subdimensions, such as in this case, the Engineering Twin, the Production Twin, and the Operation Twin. These subdimensions have proven to be suitable and helpful in the described case study, however in other applications or under different circumstances, there might be additional dimensions, which have not yet been considered.

This leads to the outlook of the presented paper. In the industrial case study, only the Engineering Twin was considered (Schweigert-Recksiek et al., 2020). Consequently, the suitability of the Production and Operation Twin still needs to be further evaluated based on the needs and possible application areas of the case study partner. Further, it should be investigated, whether the Digital Twin might add value to areas other than engineering as well. The authors of this paper see particular potential in business development. A Digital Twin should also be used for business model development. There is a continuous shift from stand-alone hardware products towards digitized product service systems. This change enables not only the improvement of product development by refining requirements, easing troubleshooting, or supporting after sales. It also gives the opportunity to understand the needs and pain points of customers and to adapt the company's offered services, payment terms, or business models respectively. Despite the application area, other subdimensions, which might additionally increase the usability of the definition by refining it, should be investigated. Especially the idea of a "Process Twin" shall mentioned. Under consideration of current challenges, it is crucial for companies to continuously adapt and improve their product development processes. Several publications have already shown the potential of engineering data for process analyses (e.g. Piccolo et al., 2018). Considering the employees working on design tasks and created documents, etc. as the physical entity, one could model a virtual real-time representation of the actually executed product development processes. Simulations and analyses applied on this model might show potentials for improvements of the process and thus influence the considered process.

\section{Acknowledgements}

The authors thank Bernd Stöhr, Louis Mahlau, and Murat Saygin for their work in elaborating the foundation of this definition, as well as Christoph Hollauer and Julian Wilberg for the collaboration in this case study.

\section{References}

Bajaj, M., Cole, B. and Zwemer, D. (2016), “Architecture To Geometry - Integrating System Models With Mechanical Design”, in SPACE Conferences and Exposition: AIAA SPACE 2016, Long Beach, California. https://doi.org/10.2514/6.2016-5470

Baltes, G. and Freyth, A. (2017), "Die radikal neuen Anforderungen unserer Zeit und die Konsequenz für Veränderungsarbeit”, in Baltes, G. and Freyth, A. (Eds.), Veränderungsintelligenz: Agiler, innovativer, unternehmerischer den Wandel unserer Zeit meistern / Guido Baltes, Antje Freyth (Hrsg.), Vol. 131, Springer Gabler, Wiesbaden, Germany, pp. 1-79. https://doi.org/10.1007/978-3-658-04889-1_1

Banerjee, A. et al. (2017), "Generating Digital Twin models using Knowledge Graphs for Industrial Production Lines", paper presented at Workshop on Industrial Knowledge Graphs, co-located with the 9th International ACM Web Science Conference, June 25-28, 2017, Troy, NY, USA. https://doi.org/10.1145/3091478.3162383

Bauernhansl, T. et al. (2016), WGP-Standpunkt Industrie 4.0, available at: https://www.ipa.fraunhofer.de/ content/dam/ipa/de/documents/Presse/Presseinformationen/2016/Juni/WGP_Standpunkt_Industrie_40.pdf (accessed 31 October 2019).

Bazilevs, Y. et al. (2015), "Isogeometric Fatigue Damage Prediction in Large-Scale Composite Structures Driven by Dynamic Sensor Data”, Journal of Applied Mechanics, Vol. 82 No. 9, p. 1149. https://doi.org/ $10.1115 / 1.4030795$ 
Blessing, L.T.M. and Chakrabarti, A. (2009), DRM, a Design Research Methodology, Springer London, London. https://doi.org/10.1007/978-1-84882-587-1

Boschert, S., Heinrich, C. and Rosen, R. (2018), "Next generation digital twin", paper presented at TMCE 2018, May 7-11, 2018, Las Palmas de Gran Canaria, Spain.

Boschert, S. and Rosen, R. (2016), "Digital Twin-The Simulation Aspect”, in Hehenberger, P. and Bradley, D. (Eds.), Mechatronic futures: Challenges and solutions for mechatronic systems and their designers / Peter Hehenberger, David Bradley, editors, Springer, Switzerland, pp. 59-74. https://doi.org/10.1007/978-3-31932156-1_5

Boureanu, L. (2017), "From Customer Service to Customer Experience: The Drivers, Risks and Opportunities of Digital Transformation”, in Klewes, J., Popp, D. and Rost-Hein, M. (Eds.), Out-thinking organizational communications: The impact of digital transformation / Joachim Klewes, Dirk Popp, Manuela Rost-Hein, editors, Management for Professionals, Vol. 4, Springer, Switzerland, pp. 145-155. https://doi.org/10. 1007/978-3-319-41845-2_11

Cadet, M. et al. (2017), "Referenzentwicklungsprozess für cybertronische Produkte und Produktionssysteme", in Eigner, M. (Ed.), Modellbasierter Entwicklungsprozess cybertronischer Systeme, Springer Berlin Heidelberg, Berlin, Heidelberg, pp. 45-62. https://doi.org/10.1007/978-3-662-55124-0_7

Eckert, C., Isaksson, O., Hallstedt, S., Malmqvist, J., Öhrwall Rönnbäck, A. and Panarotto, M. (2019), "Industry Trends to 2040", Proceedings of the Design Society: International Conference on Engineering Design, Vol. 1 No. 1, pp. 2121-2128. https://doi.org/10.1017/dsi.2019.218

Eigner, M. et al. (2019), "Definition des Digital Twin im Produktlebenszyklus", ZWF Zeitschrift für wirtschaftlichen Fabrikbetrieb, Vol. 114 No. 6, pp. 345-350. https://doi.org/10.3139/104.112107

Erikstad, S.O. (2017), "Merging Physics, Big Data Analytics and Simulation for the Next-Generation Digital Twins", in Bertram, V. (Ed.), HIPER'17: 11th Symposium on High-Performance Marine Vehicles, Zevenwacht, September 11-13, 2017, Technical University Hamburg-Harburg, Hamburg, pp. 140-150.

Grieves, M. and Vickers, J. (2017), "Digital Twin: Mitigating Unpredictable, Undesirable Emergent Behavior in Complex Systems", in Kahlen, F.-J., Flumerfelt, S. and Alves, A. (Eds.), Transdisciplinary perspectives on complex systems: New findings and approaches / Franz-Josef Kahlen, Shannon Flumerfelt, Anabela Alves, editors, Springer International Publishing, Switzerland, pp. 85-113. https://doi.org/10.1007/978-3-319-38756-7_4

Hanschke, I. (2018), Digitalisierung und Industrie 4.0 - einfach und effektiv, Carl Hanser Verlag GmbH \& Co. KG, München. https://doi.org/10.3139/9783446452992

Helu, M. and Hedberg, T. (2015), "Enabling Smart Manufacturing Research and Development using a Product Lifecycle Test Bed", Procedia Manufacturing, Vol. 1. https://doi.org/10.1016/j.promfg.2015.09.066

Huber, D. and Kaiser, T. (2015), "Wie das Internet der Dinge neue Geschäftsmodelle ermöglicht", HMD Praxis der Wirtschaftsinformatik, Vol. 52 No. 5, pp. 681-689. https://doi.org/10.1365/s40702-015-0169-6

Jones, D.E. et al. (2019), "Early Stage Digital Twins for Early Stage Engineering Design", Proceedings of the Design Society: International Conference on Engineering Design, Vol. 1 No. 1, pp. 2557-2566. https://doi.org/10.1017/dsi.2019.262

Kasote, S., Das, S. and Rao, S. (2017), “Adoption of Structural Analysis Capabilities in an IOT Based Scenario for Connected Assets”, in Bernhaupt, R., Dalvi, G., Joshi, A., Balkrishan, D.K., O’Neill, J. and Winckler, M. (Eds.), Human-computer interaction -- INTERACT 2017: 16th IFIP TC 13 International Conference, Mumbai, India, September 25-29, 2017, Proceedings. Part II / Regina Bernhaupt, Girish Dalvi, Anirudha Joshi, Devanuj K. Balkrishan, Jacki O’Neill, Marco Winckler (eds.), LNCS sublibrary. SL 3, Information systems and applications, incl. Internet/Web, and HCI, Vol. 10516, Springer, Cham, Switzerland, pp. 332335. https://doi.org/10.1007/978-3-319-68059-0_23

Kritzinger, W. et al. (2018), "Digital Twin in manufacturing: A categorical literature review and classification", IFAC-PapersOnLine, Vol. 51 No. 11, pp. 1016-1022. https://doi.org/10.1016/j.ifacol.2018.08.474

Kuhn, T. (2017), "Digitaler Zwilling”, Informatik-Spektrum, Vol. 40 No. 5, pp. 440-444. https://doi.org/ $10.1007 / \mathrm{s} 00287-017-1061-2$

Lee, J., Jin, C. and Liu, Z. (2017), "Predictive Big Data Analytics and Cyber Physical Systems for TES Systems", in Redding, L., Roy, R. and Shaw, A. (Eds.), Advances in through-life engineering services, Decision engineering, 1619-5736, Vol. 46, Springer, Cham, Switzerland, pp. 97-112. https://doi.org/ 10.1007/978-3-319-49938-3_7

Lubell, J. et al. (2013), Model-Based Enterprise Summit Report. https://doi.org/10.6028/NIST.TN.1820

Michels, J.S. (2018), "Industrial Connectivity And Industrial Analytics, Core Components of the Factory of the Future", in Sendler, U. (Ed.), The Internet of Things: Industrie 4.0 unleashed / Ulrich Sendler, editor, Vol. 2015, Springer Vieweg, Berlin, pp. 247-270. https://doi.org/10.1007/978-3-662-54904-9_15

Piccolo, S.A., Trauer, J., Wilberg, J. and Maier, A.M. (2018), "Understanding task execution time in relation to the multilayer project structure. Empirical evidence", in Leardi, C., Browning, T.R., Eppinger, S.D. and 
Becerril, L. (Eds.), DS 96: The 20th International DSM Conference, Trieste, Italy, October 15-17, 2018, Design Society, pp. 129-138.

Riesener, M. et al. (2019), "The Digital Shadow as Enabler for Data Analytics in Product Life Cycle Management", Procedia CIRP, Vol. 80, pp. 729-734. https://doi.org/10.1016/j.procir.2019.01.083

Schleich, B. et al (2017), "Shaping the digital twin for design and production engineering", CIRP Annals, Vol. 66 No. 1, pp. 141-144. https://doi.org/10.1016/j.cirp.2017.04.040

Schluse, M. and Rossmann, J. (2016), "From simulation to experimentable digital twins: Simulation-based development and operation of complex technical systems", in ISSE 2016: 2016 International Symposium on Systems Engineering Edinburgh, Scotland, George Hotel, October 3-5, 2016 proceedings papers, Edinburgh, United Kingdom, 10/3/2016 - 10/5/2016, IEEE, Piscataway, NJ, pp. 1-6. https://doi.org/10.1109/ SysEng.2016.7753162

Schroeder, G. et al. (2016), "Visualising the digital twin using web services and augmented reality", in Informatics, I.I.C.o.I. (Ed.), Proceedings, 2016 IEEE 14th International Conference on Industrial Informatics (INDIN): Palais des Congrès du Futuroscope, Futuroscope - Poitiers, France, 19-21 July, 2016, Poitiers, France, 7/19/2016 - 7/21/2016, IEEE, Piscataway, NJ, pp. 522-527. https://doi.org/10.1109/ INDIN.2016.7819217

Schuh, G. and Blum, M. (2016), "Design of a data structure for the order processing as a basis for data analytics methods", in Portland International Conference on Management of Engineering and Technology (PICMET), Honolulu, HI, USA, September 4-8, 2016, IEEE, pp. 2164-2169. https://doi.org/10.1109/ PICMET.2016.7806715

Schweigert-Recksiek, S. et al. (2020), “Conception of a Digital Twin in Mechanical Engineering: A Case Study in Technical Product Development", Design 2020 17th International Design Conference, Dubrovnik, Croatia. (accepted)

Shafto, M. et al. (2010), "Modeling, Simulation, Information Technology \& Processing”, DRAFT Technology Roadmap Area Vol. 11, Washington, DC, available at: https://www.nasa.gov/pdf/501321main_TA11MSITP-DRAFT-Nov2010-A1.pdf.

Siedlak, D.J.L. et al. (2018), “A digital thread approach to support manufacturing-influenced conceptual aircraft design”, Research in Engineering Design, Vol. 29 No. 2, pp. 285-308. https://doi.org/10.1007/s00163-0170269-0

Stark, R. and Damerau, T. (2019), "Digital Twin", in Chatti, S. and Tolio, T. (Eds.), CIRP Encyclopedia of Production Engineering, Vol. 66, Springer Berlin Heidelberg, Berlin, Heidelberg, pp. 1-8. https://doi.org/10. 1007/978-3-642-35950-7_16870-1

Stark, R., Damerau, T. and Lindow, K. (2018), "Industrie 4.0-Digital Redesign of Product Creation and Production in Berlin as an Industrial Location", in Sendler, U. (Ed.), The Internet of Things: Industrie 4.0 unleashed / Ulrich Sendler, editor, Springer Vieweg, Berlin, pp. 171-186. https://doi.org/10.1007/978-3662-54904-9_10

Stark, R., Kind, S. and Neumeyer, S. (2017), "Innovations in digital modelling for next generation manufacturing system design", CIRP Annals, Vol. 66 No. 1, pp. 169-172. https://doi.org/10.1016/ j.cirp.2017.04.045

Tao, F. et al. (2019), "Digital twin-driven product design framework", International Journal of Production Research, Vol. 57 No. 12, pp. 3935-3953. https://doi.org/10.1080/00207543.2018.1443229

Trauer, J. et al. (2020), Data Driven Engineering - Definition and Insights from an Industrial Case Study, Submitted to Nord Design 2020.

West, T.D. and Blackburn, M. (2017), "Is Digital Thread/Digital Twin Affordable? A Systemic Assessment of the Cost of DoD's Latest Manhattan Project", Procedia Computer Science, Vol. 114, pp. 47-56. https://doi.org/10.1016/j.procs.2017.09.003

Yun, S., Park, J.-H. and Kim, W.-T. (2017), "Data-centric middleware based digital twin platform for dependable cyber-physical systems”, in ICUFN (Ed.), ICUFN 2017: July 4 (Tue.)-July 7 (Fri.), 2017, Milan, Italy the Ninth International Conference on Ubiquitous and Future Networks, Milan, 7/4/2017 - 7/7/2017, IEEE, [Piscataway, NJ], pp. 922-926. https://doi.org/10.1109/ICUFN.2017.7993933 